\title{
ACOMPANHAMENTO MÉDICO VETERINÁRIO DE VACAS LEITEIRAS DE ELEVADA PRODUÇÃO, DAS RAÇAS HOLANDESA PRETA E BRANCA, VERMELHA E BRANCA E PARDO-SUÍÇA, RECÉM-PARIDAS
}

\author{
Veterinary Monitory of Height Productivity Dairy Cows, from \\ Holstein Bred, and Brown Bred in the Puerperium
}

\author{
Silvana Massuqueto \\ Médica Veterinária da PUCPR, Curitiba - PR. \\ e-mail: silvana.massuqueto@pucpr.br \\ Rodrigo de Almeida \\ Médico Veterinário, Dr., Prof. da PUCPR, Curitiba - PR. \\ e-mail: ralmeida@ufpr.br \\ Marcio Saporski Segui \\ Médico Veterinário, M. Sc., Prof. da PUCPR, Curitiba - PR. \\ e-mail: vet-maxi@vetmaxi-pec.com.br \\ Claudia Regina Vieira Rocha Coeli \\ Médica Veterinária da PUCPR, Curitiba - PR. \\ e-mail: pelos.penas@uol.com.br \\ Ivonete do R. Pereira \\ Médica Veterinária, Residente da PUCPR, São José dos Pinhais - PR. \\ e-mail: silvana.massuqueto@pucpr.br \\ Alceu Grebori \\ Médico Veterinário, Residente da PUCPR, São José dos Pinhais - PR. \\ e-mail: silvana.massuqueto@pucpr.br
}




\title{
Resumo
}

$\mathrm{Na}$ área de manejo e alimentação de vacas periparturientes, vários temas têm recebido a atenção dos pesquisadores nos últimos anos: estratégias para redução do período seco e suas conseqüências, avaliação do balanço catiônico-aniônico das dietas de vacas secas e recém-paridas, recomendações para maximizar o conforto animal no momento do parto e, finalmente, de grande relevância e grande impacto econômico, o monitoramento das vacas leiteiras recém-paridas nos primeiros dias de lactação. Em experimentos recentes, concluiu-se que vacas de maiores produções apresentaram maior incidência de enfermidades, notadamente as metabólicas, as reprodutivas e as que afetam a glândula mamária. Com o objetivo de monitorar vacas de elevada produção leiteira, 18 vacas foram acompanhadas, durante 10 dias seguidos no pós-parto, pelo diagnóstico precoce e tratamento imediato. Houve a interferência sob a forma de tratamento em 11 animais, dos quais ocorreu um óbito. Concluiu-se que por meio do diagnóstico precoce e do tratamento imediato, as desordens metabólicas podem ser minimizadas, conseqüentemente perdas econômicas associadas a essas enfermidades reduzidas, sendo o grande benefício dessa estratégia a oportunidade do monitoramento diário das vacas recém-paridas nesse delicado momento.

Palavras-chave: Manejo de vacas; Periparturiente; Vacas, Leiteiras.

\begin{abstract}
Several topics in regard to the handling and feeding of parturient cows are being considered in the last years: strategies and consequences of the reduction of the dry period, evaluation of the cationicanionic balance diets in the dry and recently delivered cows, recommendations in order to maximize the animal comfort at be parturition time and, an aspect of high relevance on the economical point of view, in regard to the monitory of the cows during the first days of lactation after the parturition day. Recent experiments pointed out towards the fact that high productive cows displayed high incidence of diseases, mainly the metabolic and the reproductive ones, as well as the diseases that affect the mammary gland. With the objective of monitoring cows of high milk productivity, the health of 18 cows were followed at the postpartum period during 10 days, through an early diagnostic and immediate treatment of incidental metabolic disorders. According to this experimental protocol, there has been the interference, on the way of treatment, in a total of 11 animals, from which 1 had died. According to this observation it is allowed to conclude that through the early diagnostic and the immediate treatment it was possible to minimize the deleterious effects of metabolic disorders and, consequently, the economical loss derived from them, in parturient cows.
\end{abstract}

Keywords: Lactating cows; Metabolic diseases; Reproductive diseases; Cows manejament.

\section{INTRODUÇÃO}

O cenário atual da pecuária leiteira, que coincide com tendências também verificadas em outras atividades agropecuárias, é a redução do número das unidades de produção (vacas leiteiras) e o aumento da produtividade (volume de leite por lactação) (HAYIRLI et al., 2002). Entretanto, esse aumento da produtividade tem sido associado a uma maior incidência de enfermidades, que podem levar a prejuízos econômicos para o produtor, um produto leite com maiores riscos de presença de resíduos de antibióticos e uma percepção negativa da atividade leiteira junto à população. O período de transição pode ser definido como o intervalo que compreende as 3 semanas pré-parto e as 3 semanas pós-parto de uma vaca leiteira. Até recentemente, manejar e alimentar vacas no período de transição não era uma grande prioridade para a indústria leiteira. Mas hoje se reconhece que a vaca passa por dramáticas alterações metabólicas e fisiológicas nesse período, em que se prepara para o parto e a futura lactação (GRUMMER, 1995). 
A diminuição da ocorrência dos efeitos do "complexo de doenças pós-parto" requer a observação e o rápido tratamento durante os dez primeiros dias a partir da data do parto (BARMORE, 2000; SALTMAN, 2001). O período pós-parto determina mudanças drásticas para o animal, incluindo mudanças nutricionais, instalações, metabolismo, demanda de energia e cálcio, condição corporal, entre outras.

Wallace e Ruegg (2000a) determinaram que a ocorrência de doenças metabólicas no período pós-parto tem um significativo efeito na ingestão de matéria seca e produção de leite no início da lactação. A média de produção determinada para os primeiros 60 dias pós-parto será mais baixa em animais que apresentem deslocamento de abomaso ou cetose. Pursley (1999) afirmou que desordens uterinas como retenção de placenta ou metrites têm impacto em futuras performances reprodutivas. A presença de mastites clínicas causa perdas econômicas de até US\$107 por episódio clínico. Esse prejuízo é causado pelo decréscimo na produção de leite e pelo descarte do leite com resíduos de antibiótico. Por esses prejuízos citados acima e outros que serão vistos no decorrer desta pesquisa, o monitoramento de vacas recém-paridas pode ser a chave para a diminuição de perdas econômicas e aumento do desempenho do rebanho leiteiro.

O principal objetivo da presente pesquisa foi o de determinar se o acompanhamento de vacas recém-paridas durante os primeiros dez dias pós-parto pode, de fato, constituir um significativo auxílio à prevenção, diagnóstico precoce e tratamento de enfermidades que têm maior incidência nas primeiras semanas pós-parto, diminuindo os custos e prejuízos e aumentando a produção leiteira e a sanidade do rebanho.

\section{MATERIAIS E MÉTODOS}

O projeto de pesquisa em vacas leiteiras no período do puerpério precoce foi realizado no complexo de Bovinocultura de Leite da Fazenda Experimental Gralha Azul (FEGA), da Pontifícia Universidade Católica do Paraná, localizada no município de Fazenda Rio Grande, Estado do Paraná, em um rebanho formado por 75 vacas leiteiras (secas e em lactação) e 25 novilhas gestantes, das raças Holandesa Preta e Branca, Vermelha e Branca e Pardo-Suíça, puras de origem. A produção desses animais situou-se na faixa de $40 \mathrm{~kg}$ leite/dia.

As atividades desenvolvidas rotineiramente na coleta de dados foram: o acompanhamento da produção de leite das vacas recém-paridas nos primeiros 10 dias de lactação, observação diária do animal após a ordenha a fim de determinar problemas de pelagem, desidratação, problemas de podologia e caminhada dos animais no retorno ao "free-stall”, para observá-los se alimentando, bebendo água e se locomovendo, medição diária da temperatura retal durante os primeiros 10 dias após o parto, independente do animal estar doente ou não, segundo a metodologia sugerida por Saltman (2001). Dados de palpação uterina diária para checar a presença de fetos, retenção de placenta ou metrite; observação diária do material fecal com objetivo de detectar a presença de sangue, diarréia ou qualquer outra anormalidade; auscultação diária do rúmen para ter a certeza do funcionamento normal, presença de gás ou deslocamento de abomaso; testes de cetose semanal no leite foram observados segundo a metodologia adotada por Geishauser, Leslie e Duffield (2000). O teste de $\mathrm{pH}$ semanal na urina, para avaliar a eficácia do balanço catiônico-aniônico da dieta, segundo a metodologia de Horst et al. (1997), além do teste California Mastitis Tests (CMT) semanalmente executado sobre o leite. Os animais observados com alguma alteração eram prontamente medicados, seja sob o aspecto preventivo, seja curativo, segundo padrões estabelecidos por Saltman (2001).

\section{RESULTADOS E DISCUSSÃO}

Do total de animais acompanhados pela Clínica (18 animais), obteve-se um percentual de 61\% $(11 / 18)$ de animais que apresentaram alterações metabólicas ou uterinas ou do aparelho digestório durante esse período (TABELA 1). 
TABELA 1 - Casuística das alterações verificadas nos animais no período de 10 dias pós-parto durante o presente estudo - Fazenda Rio Grande - PR - 2006/2007. (n=18)

Table 1 - Casuistic of the alterations found in animals at the period of 10 days postpartum during the present study - Fazenda Rio Grande - PR 2006/2007. (n=18)

\begin{tabular}{lcr}
\hline Alteracôes observadas & Animais acometidos & $\%$ \\
\hline Retençĩo de placenta & $4 / 18$ & 22,22 \\
Retençióo de placenta associada à cetose & $3 / 18$ & 16,67 \\
Cetose & $2 / 18$ & 11,11 \\
Deslocamento de abomaso & $1 / 18$ & 5,55 \\
Óbito & $1 / 18$ & 5,55 \\
\hline Total de animais acometidos & $11 / 18$ & 61,11 \\
Total de animais não acometidos & $7 / 18$ & 38,88 \\
\hline
\end{tabular}

Proporcionar manejo e alimentação adequada em vacas no período do pós-parto precoce tornou-se atualmente uma grande prioridade para a indústria leiteira. Reconhece-se que a vaca leiteira de elevada produção passa por dramáticas alterações metabólicas e fisiológicas nesse período crítico, em que a involução uterina e a atividade ovariana devem estar presentes. Para Drackley (1999), esse período corresponde à última fronteira na biologia de vacas leiteiras.

O período seco é considerado importante no ciclo de manejo do gado leiteiro. Nesse período, são observados alguns fatores de risco para a maioria das doenças no período pós-parto de vacas leiteiras, sendo que os sinais clínicos de alterações evidenciam-se após a parturição (LARSEN; MOLLER; BELLIO, 2001; WALLACE; RUEGG, 2000b). Aproximadamente, 75\% das enfermidades de gado leiteiro ocorrem nos primeiros 30 dias após o parto (LEBLANC et al., 2006).

Dentro desse contexto, observou-se taxa de retenção de placenta de 22,22\% (TABELA 1), muito além dos dados relatados por Jordan (1999), Herdt (1999) e Grohn (1999) apud Stone, Chase e Overton (1999), os quais obtiveram 9,0;12,0; 7,4\%, respectivamente. Essas três pesquisas foram aqui incluídas pela referência de Stone, Chase e Overton (1999), fato que pode ser justificável devido ao número de bezerros nascidos mortos (22,22\%), partos gemelares $(16,66 \%)$, distocia $(27,77 \%)$ e por problemas metabólicos associados (16,66\%). Nesse estudo, a cetose apresentou taxa total de 27,77\% da casuística relatada (TABELA 1), o que difere dos valores descritos por Jordan (1999), Herdt (1999) e Grohn (1999), apud Stone, Chase e Overton (1999), os quais obtiveram 3,7; 12,4; 4,6\%, respectivamente. Os corpos cetônicos são compostos primários formados do metabolismo das gorduras representados pelo b-hidroxibutirato, o acetoacetato e a acetona. Em casos de grande mobilização de gorduras, como ocorre na cetose bovina dos ruminantes, eles se acumulam no organismo, causando graves transtornos. O jejum, ou estado de anorexia de no mínimo $24 \mathrm{~h}$, pode fazer com que, principalmente vacas leiteiras em lactação com boa condição corporal, eliminem corpos cetônicos na urina (ORTOLANI, 2003). Isso pode justificar o alto índice acompanhado de cetose durante os 10 dias pós-parto, evidenciado por uma taxa de 44,44\% dos animais com redução do apetite ou anorexia.

No que se refere ao deslocamento de abomaso, a taxa registrada nesse estudo foi de 5,55\%, estando compatível com os dados citados por Jordan (1999), Herdt (1999) e Grohn (1999) apud Stone, Chase e Overton (1999), que obtiveram 3,3; 6,2; 6,3\%, respectivamente, sendo esses níveis considerados acima do aceitável (deslocamento de abomaso d" 3\%) para rebanhos leiteiros confinados e de alta produção, segundo Almeida (2003).

Houve diminuição significativa na produção leiteira em $54,54 \%$ dos animais, sendo que o restante manteve a produção constante. Após o término do tratamento, $91 \%$ dos animais monitorados apresentaram cura clínica e 9\% vieram a óbito. 


\section{CONCLUSÕES}

A principal hipótese que se verificou nesta pesquisa foi a de que a adoção de práticas nutricionais e de manejo diminui a ocorrência das principais enfermidades de vacas leiteiras, que ocorrem preferencialmente no início da lactação, por meio do diagnóstico precoce e do tratamento imediato. Com isso, certamente as elevadas perdas econômicas associadas a estas enfermidades serão minimizadas. Cabe salientar que esta metodologia não é inédita, embora seja muito pouco usada nos rebanhos brasileiros, mesmo os de alta produtividade (ALMEIDA, 2003). Em contrapartida, esta estratégia tem sido comumente adotada em rebanhos norte-americanos, notadamente os de maior tecnologia.

\section{REFERÊNCIAS}

ALMEIDA, R. Alimentação e manejo de vacas leiteiras no período de transição. Milkpoint. Seção Radares Técnicos - Nutrição. 2003. Disponível em: <www.milkpoint.com.br>. Acesso em: 01 ago. 2003.

ALMEIDA, R. Interpretação de índices sanitários, de qualidade de leite e de enfermidades. Milkpoint. Seção Radares Técnicos - Nutrição. 2006. Disponível em: <www.milkpoint.com.br.>. Acesso em: 01 out. 2006.

BARMORE, J. A. Review the basics of transition cow care. Hoard's Dairyman, Fort Atkinson, p. 769, Nov. 2000.

DRACKLEY, J. K. Biology of dairy cows during the transition period: the final frontier? Journal Dairy Science, Savoy, v. 82, p. 2259-2273, 1999.

GEISHAUSER, T.; LESLIE, K.; DUFFIELD, T. Here's a plan for dealing with ketosis. Hoard's Dairyman, Fort Atkinson, p. 397, May 252000.

GROHN, F. apud STONE, B.; CHASE, L.; OVERTON, T. Feeding high-producing herds. A supplement to Hoard's Dairyman, Fort Atkinson, v. 25, p. 2, Sept.1999.

GRUMMER, R. R. Impact of changes in organic nutrient metabolism on feeding the transition dairy cow. Journal Animal Science, Savoy, v. 73, p. 2820-2833, 1995.

HAYIRLI, A. et al. Animal and dietary factors affecting feed intake during the prefresh transition period in Holsteins. Journal Dairy Science, Savoy, v. 85, p. 3430-3443, 2002.

STONE, B.; CHASE, L.; OVERTON, T. Feeding high-producing herds. A supplement to Hoard's Dairyman, Fort Atkinson, v. 25, p. 2, Sept.1999.

HORST. R. L. et al. Strategies for preventing milk fever in dairy cattle. Journal Dairy Science, Savoy, v. 80, p. 1269-1280, 1997.

STONE, B.; CHASE, L.; OVERTON, T. Feeding high-producing herds. A supplement to Hoard's Dairyman, Fort Atkinson, v. 25, p. 2, Sept. 1999.

LARSEN, T.; MOLLER, G.; BELLIO, R. Evaluation of clinical and clinical chemical parameters in periparturient cows. Journal Dairy Science, Savoy, v. 84, p. 1749-1758, 2001.

LEBLANC, S. J. et al. Major advances in disease prevention in dairy cattle. Journal Dairy Science, Savoy, v. 89, p. 1267-1279, 2006.

ORTOLANI, E. L. Diagnóstico de doenças nutricionais e metabólicas por meio de exame de urina um ruminantes. In: SIMPÓSIO DE PATOLOGIA CLÍNICA VETERINÁRIA DA REGIÃO SUL DO BRASIL, 1., 2003, Porto Alegre. Anais... Porto Alegre: Universidade Federal do Rio Grande do Sul, 2003. p. 91-102. 
PURSLEY, R. Evaluating the reproductive performance of a dairy herd. In: CURSO NOVOS ENFOQUES NA PRODUÇÃO E REPRODUÇÃO DE BOVINOS, 3, 1999, Passos. Anais... Passos, MG, 1999. p. 47-59.

SALTMAN, R. 10 reasons to temp fresh cows for 10 days. Hoard's Dairyman West, Fort Atkinson, p. 1, July 2001.

STONE, B.; CHASE, L.; OVERTON, T. Feeding high-producing herds. A supplement to Hoard's Dairyman, Fort Atkinson, v. 25, p. 2, Sept. 1999.

WALLACE, R. L.; RUEGG, P. L. Once they calve, keep them healthy. In: COWS IN TRANSITION, MIDWEST PLAN SERVICE, 4., 2000, State. Dairy Management Seminar. Ames, IA, 01-03 Feb., p. 23-28, 2000a.

WALLACE, R. L.; RUEGG, P. Doenças metabólicas do periparto. In: CURSO NOVOS ENFOQUES NA PRODUÇÃO E REPRODUÇÃO DE BOVINOS, 4., 2000, Passos. Anais... Passos, MG, 23-25 mar., p. 6-13, 2000b.

Recebido em: 01/10/2006

Received in: 10/01/2006

Aprovado em: 30/03/2007

Approved in: 03/30/2007 